\title{
Reabilitação na síndrome do túnel do carpo
}

\author{
Carlos Michell Tôrres Santos ${ }^{1}$, Carlos Umberto Pereira²
}

\author{
Associação de Ensino e Cultura Pio Décimo e Departamento de Medicina \\ da Universidade Federal de Sergipe. Aracaju, SE, Brasil
}

\section{RESUMO}

Contexto: A síndrome do túnel do carpo é descrita como uma doença ocupacional decorrente da compressão das estruturas internas do túnel do carpo e traz alto impacto econômico à sociedade. Comprova-se que a intervenção fisioterapêutica é eficiente e também a forma mais econômica de reabilitação dessa afecção. Objetivo: O presente estudo faz uma revisão da literatura acerca dos recursos fisioterapêuticos utilizados para promover a reabilitação na síndrome do túnel do carpo.

\section{PALAVRAS-CHAVE}

Síndrome do túnel do carpo, reabilitação.

\section{ABSTRACT}

Rehabilitation in carpal tunnel syndrome

Background: The carpal tunnel syndrome is defined as an occupational pathology elapsed from compression of carpal tunnel's internal structures and results in a high economical impact to society. It has been proved that physiotherapeutic intervention is efficient and the cheapest rehabilitation form for this affection. Objective: To review the physiotherapeutic resources used to promote rehabilitation in carpal tunnel syndrome.

\section{KEY WORDS}

Carpal tunnel syndrome, rehabilitation.

\section{Introdução}

A síndrome do túnel do carpo (STC) é uma neuropatia resultante da compressão do nervo mediano em razão do espessamento do ligamento anular do carpo. ${ }^{1}$ Em consequência ao estreitamento do compartimento do túnel do carpo, ocorre aumento de resistência ao livre trânsito dos flexores dos dedos que por ali trafegam, podendo manifestar consequente parestesia, dormência e formigamento no trajeto da distribuição sensorial do nervo mediano, sinal de Tinel presente, manobra de Phalen positiva, hipoestesia, dor noturna, quadro álgico em diagramas específicos da mão e, na maioria das vezes, fraqueza da

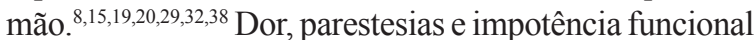
atingem primordialmente a face palmar dos $1^{\circ}, 2^{\circ}$ e $3^{\circ}$ quirodáctilos e a região tenar, principalmente o músculo oponente do polegar. ${ }^{20,28,29,33,38}$

A etiologia da STC é desconhecida; contudo, ocorre primordialmente em trabalhadores que em seu ofício desenvolvem tarefas de esforço repetitivo. ${ }^{20,28,29,33,38}$
Entre 1981 e 1991, o Departamento do Trabalho dos Estados Unidos reportou um aumento de 23.000 para 223.600 casos de desordens relacionadas ao trauma por esforço repetitivo. ${ }^{41,42} \mathrm{O}$ impacto econômico pode ser notado por meio de dados do ano de 1995, os quais demonstram que $50 \%$ de todos os trabalhadores que desenvolveram a STC perderam 30 ou mais dias de trabalho. ${ }^{5}$ Estima-se que, anualmente, um milhão de norte-americanos adultos desenvolvam a STC. ${ }^{40}$ Nesse mesmo país, os custos não médicos, relacionados à compensação pela incapacidade resultante da STC, atingem a casa dos U\$ 10.000 por mão. ${ }^{8}$ Adicionando-se os custos médicos e custos indiretos relacionados aos pacientes e a seus familiares, o custo total encontra-se entre U\$ 20.000 e U\$ 100.000 por caso. ${ }^{18}$

A terapêutica padronizada atualmente para a STC inclui, inicialmente, o tratamento conservador e, posteriormente, se necessária, a secção cirúrgica do ligamento transverso do carpo. A terapia conservadora é composta por fisioterapia, ajuste funcional do trabalho, uso de

1 Professor mestre da Associação de Ensino e Cultura Pio Décimo, Aracaju, SE.

2 Professor adjunto doutor do Departamento de Medicina da Universidade Federal de Sergipe, Aracaju, SE. 
splints e drogas anti-inflamatórias não esteroides. ${ }^{11}$ Tendo em vista todos esses aspectos, o presente estudo visa demonstrar a importância do tratamento fisioterapêutico no paciente acometido pela STC, uma vez que esse tipo de intervenção é eficiente e mais econômica. ${ }^{27,29,38}$

\section{Métodos fisioterapêuticos}

A identificação e a eliminação das condições que geram dor, tais como processo inflamatório generalizado, espasmo muscular mantido, posturas antálgicas, alterações funcionais e estruturais do aparelho locomotor, entre outras, contribuem para a eficiência da reabilitação. Para a reabilitação funcional do aparelho locomotor as medidas fisioterapêuticas são fundamentais. ${ }^{14}$ Os procedimentos fisioterapêuticos proporcionam alívio sintomático da dor, redução do uso de fármacos, diminuição da inflamação, relaxamento muscular, prevenção de deformidades, reabilitação da função motora e redução do sofrimento tecidual. ${ }^{2}$ As principais modalidades terapêuticas utilizadas no tratamento da STC são:

\section{Fase aguda}

\section{Laserterapia}

Nas duas últimas décadas, o laser de baixa potência (lasers de classe IIIb, de 5-500 mW, com feixe de luz vermelho ou próximo a infravermelho, com comprimento de onda de 600 a $1.000 \mathrm{~nm})^{27}$ tem sido utilizado no tratamento de dores associadas a condições tais como neuralgias e afecções musculoesqueléticas. ${ }^{12,21,26,36} \mathrm{Al}$ guns dos mecanismos sugeridos para o efeito terapêutico desse tipo de recurso têm sido revisados ${ }^{4,39}$ e incluem: aumento da produção de ATP pelas mitocôndrias, ${ }^{30}$ incremento do consumo de oxigênio pela célula, ${ }^{50}$ aumento dos níveis de serotonina e endorfinas, ${ }^{49}$ efeitos anti-inflamatórios ${ }^{1,25}$ e incremento da circulação sanguínea cutânea em alguns casos..$^{20,34}$ Os efeitos primários do laser de baixa potência são considerados fotobiológicos em vez de fototérmicos. ${ }^{17}$ Seu uso pode ser dado por meio da forma de aplicação convencional, diretamente sobre o local da lesão, ou por intermédio do estímulo sobre pontos de acupuntura, tendo ambas as formas excelentes resultados relatados na literatura. ${ }^{6,20,27,47,48}$

\section{Eletroterapia}

\subsection{Microcorrente TENS}

A microcorrente TENS é uma forma relativamente nova de estimulação elétrica nervosa transcutânea que é utilizada no combate da dor crônica. ${ }^{3,16,28}$ Os aparelhos de TENS convencionais utilizam a intensidade de saída da corrente em miliamperes (mA), portanto, causando uma sensação levemente incômoda no paciente, por meio de seus eletrodos de superfície. A microcorrente TENS gera a intensidade de saída da corrente em microamperes $(\mu \mathrm{A})$, fazendo com que o paciente não tenha nenhum tipo de sensação; é aplicado no tecido subcutâneo. Acredita-se que seu efeito de redução do quadro álgico, em parte, se explica por intermédio da teoria do portal. ${ }^{26}$ Observou-se que esse tipo de modalidade terapêutica aumenta as concentrações de ATP e a síntese proteica celular, com os maiores efeitos de estimulação a cerca de $500 \mu \mathrm{A}$. Correntes maiores que $5 \mathrm{~mA}$ podem deprimir as concentrações de ATP, bem como a síntese proteica e o movimento transmembrana de metabólitos. ${ }^{7}$ Sua utilização está muito mais relacionada à aplicação sobre pontos de acupuntura que sobre o local da lesão propriamente dito, tendo eficácia cada vez mais comprovada pela literatura. ${ }^{14,27}$

\subsection{TENS}

A estimulação nervosa elétrica transcutânea (TENS), utilizada por meio da aplicação de eletrodos de superfície no foco do processo álgico, é de grande valia para a diminuição da dor referida pelo paciente. A propriedade de cessação do quadro álgico é atribuída, nas formas de estimulação de baixa frequência e maior largura de pulso, à liberação de substâncias pelo sistema nervoso central, e nas formas de maiores frequências e menores períodos de pulso utilizam, para promover analgesia, o mecanismo de teoria das comportas. . $^{9} 35,43,45,46$

\section{Termoterapia}

\subsection{Termoterapia por subtração de calor}

Outra forma eficaz de se promover alívio do quadro álgico é a crioterapia. Seu efeito analgésico é proveniente da combinação da alteração da transmissão neural (que fica diminuída), redução do espasmo muscular (devida à diminuição da atividade do fuso muscular), alteração do fluxo sanguíneo aos músculos e nervos, bem como a liberação de substâncias pelo sistema nervoso central (endorfinas e encefalinas). Existem várias formas da aplicação de crioterapia, entretanto, não há indicação de que um modo particular de aplicação seja mais efetivo que outro, visto que os estudos realizados nesta área, em sua grande maioria, demonstram apenas qual forma de aplicação promove maior resfriamento tecidual em relação à outra. ${ }^{13,31,44}$

\subsection{Termoterapia por adição de calor}

$\mathrm{Na}$ fase aguda inflamatória da STC a termoterapia superficial deve ser utilizada com moderação, objetivando 
diminuir espasmos musculares e promover melhoria da circulação sanguínea local. A termoterapia profunda, por sua vez, deve ser evitada, pois pode agravar a inflamação local. ${ }^{13,31}$ No entanto, o ultrassom deve ser indicado nessa fase, em sua forma pulsátil, pois, de acordo com estudos realizados, mostra-se como um excelente recurso terapêutico no que diz respeito à diminuição do quadro álgico, à parestesia e à dor noturna. ${ }^{10,28}$

\section{Cinesioterapia}

O alongamento da musculatura envolvida nos movimentos da mão é de alta valia, pois promove uma diminuição do processo inflamatório por meio de aumento do fluxo sanguíneo local, redução da tensão e compressão das estruturas internas do túnel do carpo, o que diminui a parestesia e o quadro álgico, bem como a manutenção do arco de movimento, a qual previne o aparecimento de adesões teciduais. ${ }^{23,37} \mathrm{O}$ massageamento é um excelente método de tratamento para pacientes que apresentam dor miofascial. Deve-se levar em consideração também que esse recurso pode ser aplicado em regiões como cervical, escapular e dorsal, pois pacientes portadores da STC podem aumentar a tensão muscular dessas localidades, retardando o processo de reabilitação. ${ }^{23,31}$

\section{Fase crônica}

\section{Eletroterapia}

O enfraquecimento e a diminuição da resistência muscular podem ser revertidos por intermédio da utilização da estimulação elétrica neuromuscular (EENM). Por meio da colocação de eletrodos de superfície, os quais devem ser posicionados de modo a estimular os pontos motores musculares, é possível induzir uma contração muscular isométrica, sem qualquer esforço por parte do paciente. Esse tipo de estimulação deve seguir um programa de treinamento específico, elaborado pelo fisioterapeuta, por meio da mesclagem de parâmetros eletroterápicos. . $^{13,31,45}$

\section{Termoterapia}

Nesta fase, a termoterapia de adição de calor, nas formas de aplicação superficial e profunda, é um excelente recurso terapêutico, pois melhora o metabolismo e a circulação local, aumenta a elasticidade do tecido conectivo, relaxa a musculatura e induz a analgesia, além de preparar o segmento afetado para a aplicação da cinesioterapia e potencializando seus efeitos, uma vez que modifica as propriedades viscoelásticas do tecido conectivo por meio da redução da viscosidade e da resistência elástica tecidual. ${ }^{23} 31$

\section{Cinesioterapia}

Deve ser dada continuidade aos exercícios de alongamento, para que se faça uma preparação anterior aos exercícios ativos-livres e, posteriormente, resistidos. ${ }^{22,37}$ Assim como é primordial a preservação da amplitude de movimento, a manutenção e o ganho da força muscular se fazem metas importantes da reabilitação para que se possa promover um reequilíbrio da fisiologia musculoesquelética, uma vez que, como dito anteriormente, a limitação funcional e consequente imobilidade parcial subsequente à STC leva geralmente à perda de força e resistência muscular. ${ }^{13}$

\section{Conclusão}

Tanto o menor custo quanto a sua eficiência, demonstrada por meio da alta quantidade de recursos terapêuticos disponíveis e associáveis, tornam a fisioterapia um excelente método de reabilitação das afecções advindas da STC.

\section{Referências}

1. Ailioaie C, Lupusoru-Ailioaie LM. Beneficial effects of laser therapy in the early stages of rheumatoid arthritis onset. Laser Ther. 1999;11:79-87.

2. American Physical Therapy Association. A guide to physical therapist practice: A description of patient management. Phys Ther. 1995;75:707-64.

3. Balogun JA, Biasci S, Han L. The effects of acupuncture, electroneedling and transcutaneous electrical stimulation therapies on peripheral haemodynamic functioning. Disabil Rehabil. 1998;20:41-8.

4. Basford JR. Low-energy laser therapy: controversies and new research findings. Lasers Surg Med. 1989;9:1-5.

5. BLS Reports Rise in Cumulative Trauma. Occup Health Safety. 1995;64:14-5.

6. Branco K, Naeser MA. Carpal tunnel syndrome: clinical outcome after low-level laser acupuncture, microamps transcutaneous electrical nerve stimulation, and other alternative therapies: an open protocol study. J Altern Complement Med. 1999;5:5-26.

7. Cheng N, Van Hoof H, Bockx E, Hoogmartens MJ, Mulier JC, De Dijcker FJ, et al. The effects of electric currents on ATP generation, protein synthesis, and membrane transport in rat skin. Clin Orthop. 1982;171:264-72.

8. Dawson DM. Entrapment neuropathies of the upper extremities. N Engl J Med. 1995;329: 2013-18.

9. Deyo RA, Walsh NE, Martin DC, Schoenfeld LS, Ramamurthy $\mathrm{S}$. A controlled trial of transcutaneous electrical nerve stimulation (TENS) and exercise for chronic low back pain. N Engl J Med. 1990;322:1627-34.

10. Ebenbichler GR, Resch KL, Nicolakis P, Wiesinger GF, Uhl F, Ghanem AH, Fialka V. Ultrasound treatment for treating the 
carpal tunnel syndrome: randomised "sham" controlled trial. BMJ. 1998;316:731-5.

11. Feuerstein M, Burrell LM, Miller VI, Lincoln A, Huang GD, Berger R. Clinical management of carpal tunnel syndrome: a 12-year review of outcomes. Am J Ind Med. 1999;35:232-45.

12. Fukuuchi A, Suzuki H, Inoue K. A double-blind trial of low reactive-level laser therapy in the treatment of chronic pain. Laser Ther. 1998;10:59-64.

13. Gloth MJ, Matesi AM. Physical therapy and exercise in pain management. Clin Geriatr Med. 2001;17:525-35.

14. Guccione AA. Physical therapy for musculoskeletal syndromes. Rheum Dis Clin North Am. 1996;22:551-62.

15. Hennessey WJ, Kuhlman KA. The anatomy, symptoms and signs of carpal tunnel syndrome. Phys Med Rehabil Clin North Am. 1997:8:439-57.

16. Kahn J. Principles and practice of electrotherapy. 3.ed. New York: Churchill Livingstone; 1994. p.88-9.

17. Karu TI. Photobiological fundamentals of low-power laser therapy. IEEE J Quantum Electronics. 1987;23:1703-17.

18. Katz JN, Liang MH. Carpal tunnel syndrome and the workplace: Epidemiologic and management issues. Intern Med Specialist. 1988;9:66-73.

19. Katz JN, Larson MG, Sabra A, Krarup C, Stirrat CR, Sethi R, et al. The carpal tunnel syndrome: diagnostic utility of the history and physical examination findings. Ann Intern Med. 1990;112:321-7.

20. Katz JN, Simmons BP. Carpal Tunnel Syndrome. N Engl J Med. 2002;346:1807-12.

21. Kleinkort JA, Foley RA. Laser acupuncture: its use in physical therapy. Am J Acupunct. 1984;12:51-6.

22. Kowal MA. Review of physiological effects of cryotherapy. J Orth Sports Phys Ther. 1982;5:66-73.

23. Madaleno JA, Uhl TL. Rehabilitation concepts and supportive devices for overuse injuries of the upper extremities. Clin Sports Med. 2001;20:621-39.

24. Masear VR, Hayes JM, Hyde AG. An industrial cause of carpal tunnel syndrome. J Hand Surg. 1986;11A:222-7.

25. Mester E, Mester AF, Mester A. The biomedical effects of laser application. Lasers Surg Med. 1985;5:31-9.

26. Melzack R, Wall PD. Pain mechanisms: a new theory. Science. 1965;150:971-9.

27. Naeser MA, Hanh KK, Lieberman BE, Branco KF. Carpal tunnel syndrome pain treated with low-level laser and microamperes transcutaneous eletric nerve stimulation: a controlled study. Arch Phys Med Rehabil. 2002;83:978-88.

28. Oztas O, Turan B, Bora I, Karakaya MK. Ultrasound therapy effect in carpal tunnel syndrome. Arch Phys Med Rehabil. 1998;79:1540-4.

29. Pádua L, Pádua R, Aprile I, D’Amico P, Tonali P. Carpal tunnel syndrome: relationship between clinical and patient-oriented assessment. Clinical Orthop Related Res. 2002;2002:128-34.

30. Passarella S. He-Ne laser irradiation of isolated mitochondria. J Photochem Photobiol. 1989;3:642-3.

31. Rakel B, Barr JO. Physical modalities in chronic pain management. Nurs Clin North Am. 2003;38:477-94.

32. Rempel D, Dahlin L, Lundborg G. Pathophysiology of nerve compression syndromes: response of peripheral nerves to loading. J Bone Joint Surg (Am). 1999;81:1600-10.

33. Roquelaure $Y$, Mechali S, Dano C, Fanello S, Benetti $F$, Bureau $D$, et al. Occupational and personal risk factors for carpal tunnel syndrome in industrial workers. Scand J Work Environ Health. 1997;23:364-9.

34. Schindl A, Schindl M, Schon H, Knobler R, Havelec LO, Schindl L. Low-intensity laser improves skin circulation in patients with diabetic microangiopathy. Diabetes Care. 1998;21:390-4.

35. Sonde L, Gip C, Fernaeus SE, Nilsson CG, Viitanen M. Stimulation with low frequency $(1.7 \mathrm{~Hz})$ transcutaneous electric nerve stimulation (low-tens) increases motor function of the post-stroke paretic arm. Scand J Rehabil Med. 1998;30:95-9.

36. Soriano F, Rios R. Gallium arsenside laser treatment of chronic low back pain: a prospective, randomized and double blind study. Laser Ther. 1998;10:175-80.

37. Spring H, Schneider W, Tritschler T. Stretching. Orthopade. 1997;26:981-6.

38. Szabo RM. Carpal Tunnel Syndrome as a repetitive motion disorder. Clin Orthop Related Res. 1998;351:78-89.

39. Tam G. Low power laser therapy and analgesic action. J Clin Laser Med Surg. 1999;17:29-33.

40. Tanaka S, Wild DK, Seligman PJ, Behrens V, Cameron L, Putz-Anderson V. The US prevalence of self-reported carpal tunnel syndrome: 1988 national health survey data. Am J Pub Health. 1994;84:1846-8.

41. US Department of Labor, Bureau of Labor Statistics. Occupational injuries and illnesses in the United States by injury. 1981. Washington (DC): US Government Printing Office; 1983.

42. US Department of Labor, Bureau of Labor Statistics. Occupational injuries and illnesses in the United States by injury. 1991. Washington (DC): US Government Printing Office; 1993.

43. Van der Spank JT, Cambier DC, De Paepe HM, Danneels LA, Witvrouw EE, Beerens L. Pain relief in larbour by transcutaneous electrical nerve stimulation (TENS). Arch Gynecol Obstet. 2000;264:131-6.

44. Walker J. Relief from chronic pain by low power laser irradiation. Neurosci Lett. 1983;43:339-44.

45. Watson T. The role of electrotherapy in contemporary physiotherapy practice. Man Ther. 2000;5:132-41.

46. Weiner DK, Rudy TE, Glick RM, Boston JR, Lieber SJ, Morrow LA, Taylor S. Efficacy of percutaneous electrical nerve stimulation for the treatment of chronic low back pain in older adults. J Am Geriatr Soc. 2003;51:599-608.

47. Weintraub MI. Noninvasive laser neurolysis in carpal tunnel syndrome. Muscle Nerve. 1997;20:1029-31.

48. Wong E, Lee G, Zucherman J, Mason DT. Successful management of female office workers with "repetitive stress injury" or "carpal tunnel syndrome" by a new treatment modality: application of low level laser. Int J Clin Pharmacol Ther. 1995;33:208-11.

49. Yamamoto H, Ozaki A, Iguchi N, Kinoshita S. Antinociceptive effects of laser irradiation of Hoku point in rats. Pain Clin. 1988;8:43-8.

50. Yu W, Naim JO, McGowan M, Ippolito K, Lanzafame RJ. Photomodulation of oxidative metabolism and electron chain enzymes in rat liver mitochondria. Photochem Photobiol. 1997;66:866-71.

Original recebido em junho de 2009

Aceito para publicação em setembro de 2009

Endereço para correspondência

Prof. MSc. Carlos Michell Tôrres Santos

Av. Farmacêutica Cezartina Regis Lebre, 134, Conjunto Sol Nascente

49095-100 - Aracaju, SE

E-mail:michellfisio@hotmail.com.br 